\title{
Reproducibility of neutron activated Sm-153 oral dose formulations intended for human administration
}

Type: Article

\section{Abstract:}

Neutron activation of Sm-152 offers a method of radiolabeling for the in vivo study of oral dose formulations by gamma scintigraphy. Reproducibility measurements are needed to ensure the robustness of clinical studies. 204 enteric-coated guaifenesin core tablets (10 mg of $\mathrm{Sm}(2) \mathrm{O}(3)$ ) were irradiated by thermal neutrons to achieve $1 \mathrm{MBq}$ at $48 \mathrm{~h}$. Administered activities were $0.86+/-0.03 \mathrm{MBq}$. Good reproducibility (CV=3.5\%) was observed over 24 weeks ensuring that volunteer doses were within the dose reference level of $0.8 \mathrm{mSv}$.

\begin{tabular}{|c|c|}
\hline Author & $\begin{array}{l}\text { - Yeong, C. H. } \\
\text { - Blackshaw, P. E. } \\
\text { - Ng, K. H. } \\
\text { - Abdullah, B. J. J. } \\
\text { - Blaauw, M. } \\
\text { - Dansereau, R. J. } \\
\text { - Perkins, A. C. }\end{array}$ \\
\hline Source & Applied Radiation and Isotopes \\
\hline ISSN & 0969-8043 \\
\hline $\mathrm{DOI}$ & 10.1016/j.apradiso.2011.04.017 \\
\hline Volume (Issue) & $69(9)$ \\
\hline Page & 1181-1184 \\
\hline Year & 2011 \\
\hline
\end{tabular}

Keyword:

Gamma scintigraphy, Neutron activation, Oral dosage formulation, Reproducibility, Sm153, gamma-scintigraphic evaluation, nuclear-medicine, drug-delivery, dosage, forms, tablets, capsules, colon

Please Cite As:

YEONG, C. H., BLACKSHAW, P. E., NG, K. H., ABDULLAH, B. J. J., BLAAUW, M., DANSEREAU, R. J. \& PERKINS, A. C. 2011. Reproducibility of neutron activated Sm-153 oral dose formulations intended for human administration. Applied Radiation and Isotopes, 69, 1181-1184. 
URL:

- $\quad$ http://apps.webofknowledge.com search via Accession No >> 000292357500004

- $\quad$ http://www.scopus.com/inward/record.url?eid=2-s2.079957601379\&partnerID=40\&md5=ee3eb68a2cbb3be543d8a0b3954adb30

- $\quad$ http://www.ncbi.nlm.nih.gov/pubmed/21550260

- http://www.sciencedirect.com/science/article/pii/S096980431100251X

- $\quad$ http://scienceindex.com/stories/1611878/Reproducibility of neutron activated S m153 oral dose formulations intended for human administration.html\#.UHtV BcXMhoc

- $\quad$ http://lib.bioinfo.pl/paper:21550260 